\title{
Hybrid Macro- and Generalized Selection Combining Microdiversity in Lognormal Shadowed Rayleigh Fading Channels
}

\author{
A. Adinoyi and H. Yanikomeroglu \\ Broadband Communications and Wireless Systems (BCWS) centre \\ Dept. of Systems and Computer Engineering \\ Carleton University, Canada \\ Email: adinoyi,halim@sce.carleton.ca
}

\author{
S. Loyka \\ School of Information Technology and Engineering \\ University of Ottawa, Canada \\ Email: sloyka@site.uottawa.ca
}

\begin{abstract}
The performance of hybrid micro-diversity, in the form of generalized selection combining (GSC), and macrodiversity is presented for lognormal shadowed Rayleigh fading channels. The GSC-augmented macrodiversity consists of $K$ ports in a cell site, each port carrying $N$ microscopic diversity antennas. The macroscopic diversity involves selecting the port with the highest long-term local mean SNR among the $\mathrm{K}$ ports, and the GSC uses $n$ strongest signals of the $N$ branch received signals from that port for processing. We derive analytical expressions for error probability and outage for systems employing this hybrid scheme. The expressions are valid for any configurations of $K, N, n$. In microcell systems substantial correlation could exist among the ports in contrast to the zero correlation assumption of macrocell; results are also shown for correlated lognormal shadowed Rayleigh channels. Extensive simulation is carried out to validate the analytical expressions derived.
\end{abstract}

\section{INTRODUCTION}

In this paper, a performance analysis of a system employing micro-diversity, in the form of generalized selection combining (GSC) on top of macro-diversity is presented. GSC introduced in [1] is a hybrid of maximal ratio combining (MRC) and selection combining (SC) and expanded upon in [2]. In a GSC receiver the signals are ordered and a subset is selected and treated as in MRC. The advantages of GSC over MRC and SC include moderate receiver complexity, lower electronic cost and exemption of weak signals from the combining. These weak signals are prone to channel estimation errors.

In cellular networks, large-scale fading known as shadowing can put a heavy limit on system performance. Shadowing is caused by obstruction of buildings, trees and other man-made structures. The signal can suffer a tremendous loss and could cause the local mean level to fluctuate about the area mean. This variation is well modeled by lognormal distribution with an area mean $\mu_{k} \mathrm{~dB}$ and standard deviation $\sigma_{k} \mathrm{~dB}$, a parameter that describes the extend of shadowing. In urban cities $\sigma_{k}$ can be as high as $12 \mathrm{~dB}$.

Microscopic diversity, unfortunately cannot combat shadowing. In such situations, diversity achieved through closely spaced antennas will be penalized heavily, as all the antennas will suffer from the same detrimental effects of shadowing.

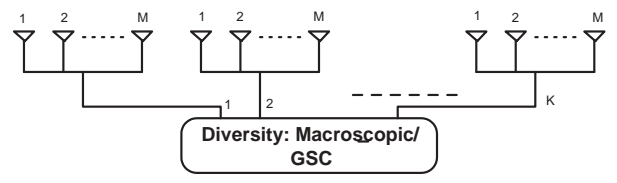

Fig. 1. Antenna structure.

Macro-diversity is used to alleviate the effects of shadowing, where multiple signals are received at $K$ widely located radio ports, ensuring that different long-term fading is experienced by these signals. Widely separated antennas referred to as distributed and sectorized distributed antenna system have been used to improve performance of communications system [3] and MRC on the distributed antenna was studied in [4], although this idea had already been discussed in [5]. Outage probabilities and capacity in the context of CDMA systems are the performance measures considered in [3] and [4].

This present work is different in the following way. This paper presents symbol error probability (SEP) expressions for modulation schemes employing the GSC-augmented macrodiversity. The macroscopic diversity selects the radio-port with least long-term shadowing effect and of the $N$ signal available $n$-strongest are selected for combining. We derive analytical expressions for error rate and outage for systems employing this hybrid scheme. The expressions are valid for any arbitrary configurations of $K, N, n$. In the classical diversity schemes, system performance criteria like diversity order and asymptotic behaviour (a logical limit) have been adequately quantified, composite fading (macro- and microdiversity), unfortunately, does not easily lend itself amenable to these limits. Efforts are on-going at providing a quantitative analysis for this scheme in the lights of these logical limits.

\section{SYSTEM AND CHANNEL DESCRIPTION}

Fig. 1 shows the proposed antenna scheme. In the network, each area is equipped with $K$ widely separated ports with each port carrying $N$ microdiversity antennas. We have assumed, following the sectorized distributed antenna system proposed 
in [3], that a separate feeder exists for each signal to transport it to a central unit where processing is carried out. Hence, a total of $N \times K$ feeders are required. The channel is modeled as Rayleigh flat fading with lognormal shadowing and additive white Gaussian noise (AWGN). It has been assumed that these two fading processes are independent. We have considered uncorrelated as well as correlated shadowing.

For the $K$-port cellular system and with each port equipped with $N$-branch micro-diversity antennas, $\left\{\alpha_{k, l}\right\}, 1 \leq k \leq K$ and $1 \leq l \leq N$, represent the multiplicative distortions or fades suffered by the signal in that path. They are independent and identically distributed Rayleigh random variables with a common mean square $E\left[\alpha_{k, l}^{2}\right]=\Omega_{k}$. Therefore, the local mean received signal-to-noise ratio per branch is $\frac{E_{s}}{N_{0}} \Omega_{k}$, where $E_{s}$ is the signal energy and $N_{0}$ is the single-sided power spectral density of the AWGN. $\Omega_{k}$ is assumed to be from lognormal distribution, that is $G_{k}=10 \log _{10}\left(\Omega_{k}\right)$ has Gaussian distribution with a certain area mean $\mu_{k} \mathrm{~dB}$ and variance $\sigma_{k} \mathrm{~dB}$. Consider that $G^{*}=\max \left\{G_{1}, G_{2}, \cdots, G_{K}\right\}$ is the local mean value which corresponds to the port selected for the macroscopic diversity and observe that the $\Omega^{*}=10^{0.1 G^{*}}$. Since we assume statistical independence and identical distribution, the pdf of $\mathrm{G}^{*}$ can be written [8] as

$$
\begin{aligned}
p_{G^{*}}(g, K) & =\frac{K}{\sqrt{2 \pi \sigma^{2}}} \exp \left(-\frac{(g-\mu)^{2}}{2 \sigma^{2}}\right) \\
& \times\left[1-\frac{1}{2} \operatorname{erfc}\left(\frac{g-\mu}{\sqrt{2 \sigma^{2}}}\right)\right]^{K-1},
\end{aligned}
$$

where for tractable analysis we have assumed that $\mu_{k}=\mu$ and $\sigma_{k}=\sigma, k=1, \cdots, K$, and $g$ is a real number in the interval $(-\infty, \infty)$.

\section{ERror Rate CALCUlations}

The symbol error probability (SEP) for MPSK modulation conditioned on the fade samples can be expressed for the GSC as,

$$
P_{m p s k}\left(\left.e\right|_{\beta, g}\right) \approx h \times \operatorname{erfc}\left(\sqrt{\beta \bar{\gamma} \sin ^{2}\left(\frac{\pi}{\mathrm{M}}\right) \frac{E_{s}}{N_{0}}}\right)
$$

where for BPSK, $h=1 / 2$ which yields an equality in (2) and for higher MPSK constellations, $h=1$. MPSK has been used as an illustrative example. Coherent modulation schemes having error rate defined by $\mathrm{Q}$ function in AWGN can be accommodated in the analysis presented here.

Let us define $d=\sin ^{2}\left(\frac{\pi}{M}\right) \frac{E_{s}}{N_{0}}$ and note that $\beta$ represents the total of the combined fading branches. This is given as $\beta=\sum_{i=1}^{n} \alpha_{i}^{2}$, and $\bar{\gamma}=10^{g / 10}$, where $\alpha$ 's represent the microscopic channel gains, and $n$ represents the number of branches selected from the microdiversity branches available. Equation (2) implies that the error performance is conditioned on macroscopic parameter $G^{*}$ and to obtain the performance two pdf averagings are required. Specifically, we need to evaluate

$$
\begin{aligned}
P_{M} & =\int_{0}^{\infty} P_{m p s k}\left(\left.e\right|_{\beta, g}\right) \int_{-\infty}^{\infty} p\left(\beta ;\left.n\right|_{g}\right) p_{G^{*}}(g, K) d g d \beta \\
& =\int_{-\infty}^{\infty} p_{G^{*}}(g, K) d g \underbrace{\int_{0}^{\infty} p\left(\beta ;\left.n\right|_{g}\right) P_{m p s k}\left(\left.e\right|_{\beta, g}\right) d \beta}_{I_{2}} .
\end{aligned}
$$

For the ordered fade amplitudes, $\alpha_{1}, \alpha_{2}, \cdots, \alpha_{N}$, the pdf $p(\beta)$ of the $n$ largest can be expressed using the results in [2], where we have exploited the independence of the microscopic and macroscopic fades,

$$
\begin{aligned}
p(\beta)=\left(\begin{array}{c}
N \\
n
\end{array}\right)\left[\frac{\beta^{n-1} e^{-\beta}}{(n-1) !}+\sum_{l=1}^{N-n}(-1)^{n+l-1}\left(\begin{array}{c}
N-n \\
l
\end{array}\right)\right. \\
\times\left(\frac{n}{l}\right)^{n-1} e^{-\beta}\left(e^{-\frac{l \beta}{n}}-\sum_{p=0}^{n-2} \frac{1}{p !}\left(-\frac{l \beta}{n}\right)^{p}\right) .
\end{aligned}
$$

Using (4), we can express $I_{2}$ in a form to allow the use of the following equality [9]

$$
\begin{gathered}
\frac{1}{2(W-1) !} \int_{0}^{\infty} \operatorname{erfc}(\sqrt{x y}) e^{-y(1-x)} y^{W-1} d y= \\
\frac{1}{2^{2 W}} \sum_{j=1}^{W}\left(\begin{array}{c}
2 W-j-1 \\
W-1
\end{array}\right)\left(\frac{2}{1+\sqrt{x}}\right)^{j} .
\end{gathered}
$$

Let $\eta_{1}(l, n, N)=(-1)^{n+l-1}(n / l)^{n-1}\left(\begin{array}{c}N-n \\ l\end{array}\right)$, then, the expression for the SEP of MPSK conditioned on the lognormal fading can be expressed as $P_{m p s k}\left(\left.e\right|_{g}\right)=$

$$
\begin{aligned}
& \left(\begin{array}{c}
N \\
n
\end{array}\right) \frac{1}{2^{2 n-1}} \sum_{j=1}^{n}\left(\begin{array}{c}
2 n-j-1 \\
n-1
\end{array}\right)\left(\frac{2}{1+\sqrt{\delta}}\right)^{j} \frac{1}{(1+\bar{\gamma} d)^{n}} \\
& +\left(\begin{array}{c}
N \\
n
\end{array}\right) \sum_{l=1}^{N-n} \eta_{1}(l, n, N) \frac{1}{(l / n+1+\bar{\gamma} d)} \frac{1}{\left(1+\sqrt{\delta_{2}}\right)} \\
& \quad-\left(\begin{array}{c}
N \\
n
\end{array}\right) \sum_{l=1}^{N-n} \eta_{1}(l, n, N) \sum_{p=0}^{n-2}(-l / n)^{p} \\
& \times \frac{2}{[4(1+\bar{\gamma} d)]^{p+1}} \sum_{j=1}^{p+1}\left(\begin{array}{c}
2(p+1)-j-1 \\
p
\end{array}\right)\left(\frac{2}{1+\sqrt{\delta}}\right)^{j}
\end{aligned}
$$

where, $\delta=\frac{\bar{\gamma} d}{1+\bar{\gamma} d}, \delta_{2}=\frac{\bar{\gamma} d}{1+\bar{\gamma} d+\frac{l}{n}}$. Finally, the error probability can be obtained by evaluating the integral in (4), that is

$$
P_{M}=\int_{-\infty}^{\infty} P_{m p s k}\left(\left.e\right|_{g}\right) p_{G^{*}}(g, K) d g
$$

The integral in (7) can be easily evaluated using numerical integration technique available in most standard mathematical packages. The closed form for it, however, remains an open question.

\section{EFFECT OF BRANCH CORRELATION}

The increasing demand for digital cellular services calls for migration from macrocell systems towards microcell and picocell systems. Hence, uncorrelated assumption that has given over-estimation in macrodiversity gains (see results below) can no longer be valid. Due to cell size in the microcell and picocell systems, significant correlation may exist among macrodiversity ports. The zero correlation case therefore serves as a lower bound for the performance. The 
effect of branch correlation has been studied for Rician fading and correlated macrodiversity for MRC microscopic diversity [6]. It is concluded that branch correlation cannot be ignored in the performance analysis of small cell systems. For the analysis of correlated macroscopic branches, the pdf of $G^{*}$ in (1) has to be modified. For a 2-branch diversity the pdf can be expressed as

$$
\begin{aligned}
p_{G^{*}}(g) & =\sqrt{\frac{2}{\pi \sigma^{2}}} \exp \left(-\frac{(g-\mu)^{2}}{2 \sigma^{2}}\right) \\
& \times\left[1-\frac{1}{2} \operatorname{erfc}\left(\sqrt{\frac{1-\rho}{1+\rho}}\left(\frac{g-\mu}{\sqrt{2 \sigma^{2}}}\right)\right)\right],
\end{aligned}
$$

where $\rho$ is the correlation coefficient.

\section{Outage Probability Calculation}

Outage is defined in terms of certain link quality measure becoming worse than a preset threshold value. This link quality could be the instantaneous or local mean SNR or the corresponding error rate. A number of work has considered either of this performance measure. For example [6] used instantaneous SNR while [8] considered the bit error rate as the quality criterion. Since most researchers have considered outage probability, we also present performance expression for calculating the probability that the instantaneous error rate exceeds a certain threshold for a stationary user in the macroscopic and GSC diversity architecture. Bit error probability is used here as the outage criterion. This outage analysis will be considered quasi-static, as against the mobile outage, in the sense that the user will be assumed to be stationary at the point the average outage is evaluated. However, this can easily be extended to mobile outage as well.

The instantaneous SNR can be expressed as $\beta\left(10^{g / 10}\right) \frac{E_{s}}{N_{0}}$ and hence, the corresponding conditional error rate for QPSK, $P\left(\left.e\right|_{\beta, g}\right)$, written as $P_{e}$ for compactness is,

$$
P_{e}=1 / 2 \times \operatorname{erfc}\left(\sqrt{\beta 10^{g / 10} E_{b} / N_{0}}\right) .
$$

The probability that $P_{e}$ is greater than a set threshold, say $\zeta$ is equivalent to having a SNR level $\Gamma$ less than the required $\mathrm{SNR}$ is given as,

$$
\operatorname{Pr}\left(\Gamma<P_{e}^{-1}(\zeta)\right)=\operatorname{Pr}(\Gamma<\varepsilon)
$$

where $\varepsilon$ represents the corresponding threshold SNR for the required error rate $\zeta$, i.e $P_{e}(\varepsilon)=\zeta$. Notice that $\Gamma=$ $10^{g / 10} \beta \frac{E_{b}}{N_{0}}$. Now, using the information in (9) and (10), we can express the threshold $\beta_{t h r}$ as $\beta_{t h r}=\frac{\left[\operatorname{erfc}^{-1}(2 \zeta)\right]^{2}}{10^{\frac{g}{10}} \frac{E_{b}}{N 0}}$. With this representation, the quasi-static outage probability can be obtained by averaging over the spatial environment,

$$
\begin{aligned}
P_{\text {outage }} & =\int_{0}^{\beta_{t h r}}\left[\int_{-\infty}^{\infty} p\left(\beta ;\left.n\right|_{g}\right) p_{G^{*}}(g, K) d g\right] d \beta, \\
& =\int_{-\infty}^{\infty} p_{G^{*}}(g, K) d g \underbrace{\int_{0}^{\beta_{t h r}} p\left(\beta ;\left.n\right|_{g}\right) d \beta}_{I_{3}} .
\end{aligned}
$$

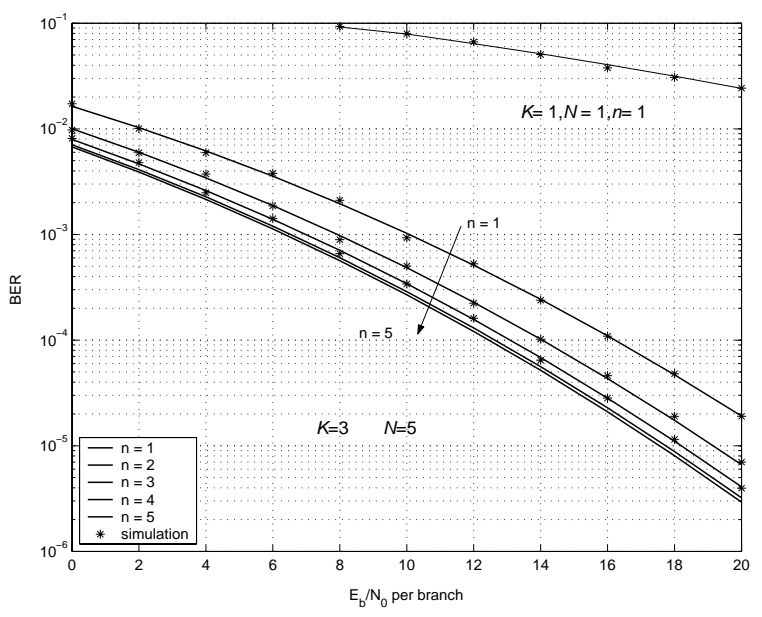

Fig. 2. Performance of BPSK in macro-diversity and GSC in lognormal shadowing $(\sigma=12 \mathrm{~dB})$ and Rayleigh fading.

Using the pdf given in (4), we can express the integral denoted above as $I_{3}$ in this form

$$
\begin{aligned}
I_{3} & =\left(\begin{array}{c}
N \\
n
\end{array}\right)\left[1-e^{-\beta_{t h r}} \sum_{k=0}^{n-1} \frac{\beta_{t h r}^{k}}{k !}+\sum_{l=1}^{N-n} \eta_{1}\right. \\
& \left.\times\left(\frac{1-e^{-\beta_{t h r}\left(\frac{l+n}{n}\right)}}{\frac{n+l}{n}}-\sum_{p=0}^{n-2} \eta_{2}\left(1-e^{-\beta_{t h r}} \sum_{t=0}^{p} \frac{\beta_{t h r}^{t}}{t !}\right)\right)\right]
\end{aligned}
$$

where $\eta_{2}(l, n, p)=\left(\frac{-l}{n}\right)^{p}$. Using (11) and (12) the average outage probability can be evaluated.

Notice that the outage for other modulation techniques can similarly be obtained. The only effort that is required is how to obtain an expression for the threshold on the $\beta$, The direction of work being developed in [7] will be very helpful in obtaining this threshold level.

\section{DISCUSSION OF RESULTS}

Some illustrative numerical results are presented for the GSC-augmented macroscopic diversity using the expressions derived above. Fig. 2 shows the BER curves for BPSK modulation scheme employed with this hybrid scheme in uncorrelated lognormal shadowing and Rayleigh flat fading for the antenna configuration $K=3, N=5, n=1, \cdots, N$. The variance of the lognormal shadowing $\sigma$ is set at $12 \mathrm{~dB}$. The results provided are for a mean of zero $\mathrm{dB}$ assumption for the lognormal fading, which in effect, removes the distancedependent received power variations. In other words, the performance is evaluated at locations equidistant from the macro-diversity ports. The simulated results are denoted by (*) and is observed that analytical performance agree perfectly with the simulations. Fig. 3 shows the performance for BPSK modulation with antenna configuration $K=2, N=5$ and for all possible values of selection order, $1 \leq n \leq N$, and in this case, $\sigma$ is set to $8 \mathrm{~dB}$. The simulation results are also plotted and denoted by $(*)$, which agree well with the derived expression. It is interesting to observe that there seems to be fixed gain in SNR of about $2 \mathrm{~dB}$ for the system when $n$ is 


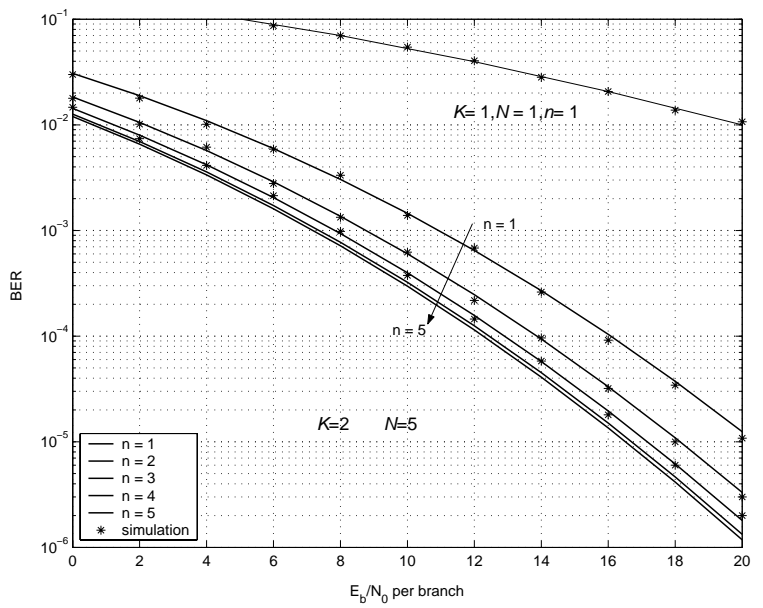

Fig. 3. Performance of BPSK in macro-diversity and GSC in lognormal shadowing $(\sigma=8 \mathrm{~dB})$ and Rayleigh fading.

increased from one to two for all the number of ports $\mathrm{K}$ and microscopic diversity branches $\mathrm{N}$ considered. This can easily be deduced from Figs. 2 - 3. This $2 \mathrm{~dB}$ gain does not seems to be affected by the amount of lognormal shadowing. However, there is a diminishing returns as $n$ continue to increase. In these figures, a $1 \mathrm{~dB}$ gain is generally observed when $n=3$ over $n=2$. The same trend is observed for the extensive combination of the antenna elements we have tried. In all, smulations are also conducted to validate the analytical results. We also observed that when $N=5$, for example, $n=4$ has already given almost all the diversity gain. Therefore, extra cost of processing and electronics could be saved provided we know when adding more branches does not give any appreciable returns. In Figs. 2 and 3 the performance curves are also shown for the case where no diversity is used. The huge gain of the combined diversity schemes over the the single channel case is very evident.

As mentioned earlier, in microcells and picocells uncorrelatedness assumption might not be strictly valid. If uncorrelatedness is assumed it does lead to over-estimation in the gains from macrodiversity.

The results presented in Figs. 4 and 5 show the detrimental effect of correlation among the branches. For example for a target error rate of $10^{-3}$ and correlation coefficient $\rho=0.5$ and in all the $n$ order microscopic combining, $4.5-6 \mathrm{~dB}$ more SNR is required in comparison with no correlation. The margin widens for increased correlation coefficient. Simulation results in all cases match the analysis closely. Looking at these figures, a confirmation of intuitive reasoning is at once observed for the case of $\rho=1.0$, completely correlated branches. In words, no new information is provided when more of the same kind of items are observed. We can see therefore, that selecting $n=2$ (Fig. 4) or selecting $n=4$ (Fig. 5) gives almost the same error rate. In general, the results given in these two figures and others not shown point to the well known fact that diversity alone is not an efficient technique to combat correlated fading.

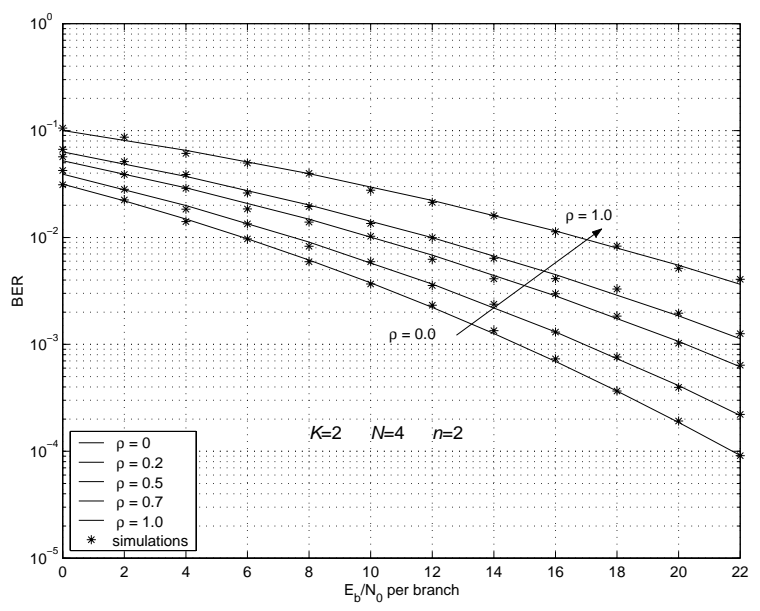

Fig. 4. Performance of BPSK in correlated 2-branch macro-diversity, $\sigma=12$ $\mathrm{dB}, n=2$.

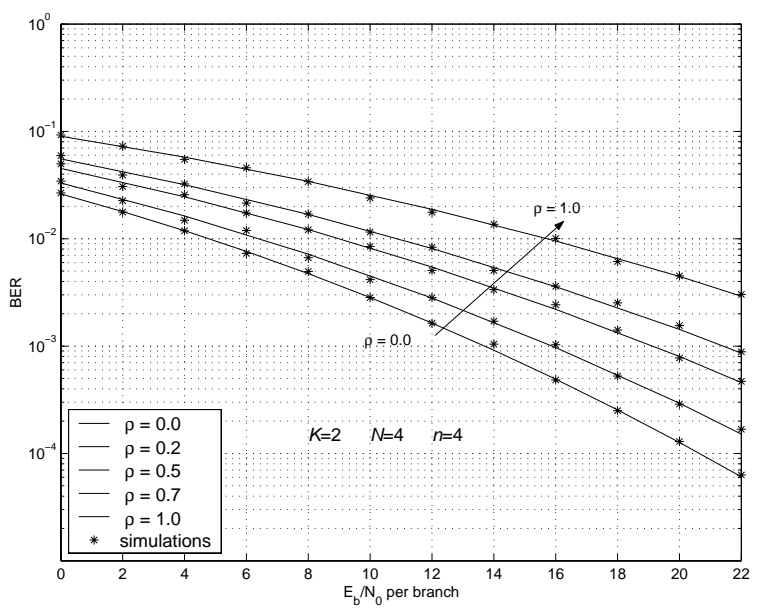

Fig. 5. Performance of BPSK in correlated 2-branch macro-diversity, $\sigma=12$ $\mathrm{dB}, n=4$.

Next, numerical results are presented for the outage probability. The curves in Fig. 6 show the outage probability versus threshold bit error rate of QPSK obtained using the expressions in (11) and (12). Different values of macro-diversity ports $K$, micro-diversity branches $N$ and generalized selection order $n$ are used with different values of branch $E_{b} / N_{0}$. Lognormal shadowing variance $\sigma=6 \mathrm{~dB}$ is considered. From previous results, we note that to attain low error rates we require very large $E_{b} / N_{0}$ in channel subjected to fading and shadowing and have seen how combination of micro-diversity and macrodiversity can be used to reduce this power requirements. For the outage curves, we also observe that for a given error-rate threshold, the amount of reduction of outage increases with the number diversity branches.

\section{CONCLUSION}

Performance analysis expressions have been presented for a generalized selection combining (GSC) augmnented macroscopic diversity in lognormal shadowing and Rayleigh fading 


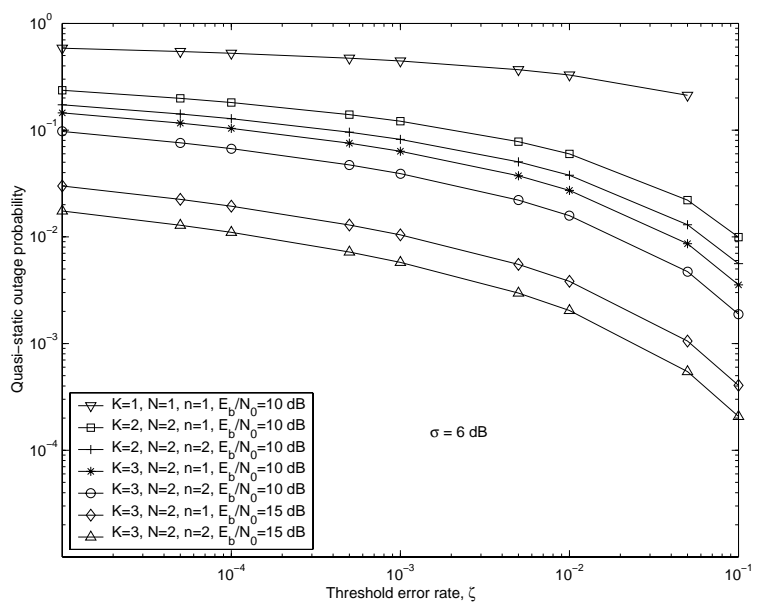

Fig. 6. Outage probability of K macro-diversity and GSC $(\mathrm{N}, \mathrm{n})$ in lognormal shadowing $(\sigma=6 \mathrm{~dB})$ and Rayleigh fading.

environments. Each of the $K$ macroscopic port is equipped with $N$ microscopic diversity antennas. The macroscopic diversity involves selecting the port with the highest longterm local mean SNR among the $\mathrm{K}$ ports, and the GSC uses $n$ strongest signals of the $N$ branch received signals from that port for processing. We assumed the feasibility of sectorized distributed system where the signals from all the ports can be transported to a central processing unit. We derive analytical expressions for error rate for systems employing this hybrid scheme. The expressions are valid for any configurations of $K, N, n$. Expressions are also presented for evaluating the outage probability for a stationary mobile user. Finally, extensive simulations are used to validate the derived expressions.

\section{ACKNOWLEDGEMENT}

Thanks to

\section{REFERENCES}

[1] N. Kong and L. B. Milstein, "Combined average SNR of a generalized diversity selection combining scheme", Proc. of IEEE Int'l. Conf. on Commun., pp. 1556-1560, June 1998.

[2] M-S. Alouini and M. K. Simon, "An MGF-based performance analysis of generalized selection combining over Rayleigh fading channels", IEEE Trans. on Commun., 48(3), pp. 401-415, March 2000.

[3] H. Yanikomeroglu and E. S. Sousa, "CDMA sectorized distributed antenna system", IEEE International Symposium on Spread Spectrum Techniques and Applications, pp. 792-797, September 1998.

[4] W. Roh and A. Paulraj, "Outage performance of the distributed antenna systems in a composite fading channel", IEEE Vehic. Techn. Conf., pp. 1520-1524, 2002.

[5] W.-P Yung, "Probability of bit error for MPSK modulation with diversity reception in Rayleigh fading and log-normal shadowing channel", IEEE Trans. on Commun., 38(7), pp. 933-937, July 1990.

[6] L.-C. Wang, G. L. Stuber and C.-T. Lea, "Effects of Rician fading and branch correlation on a local-mean-based macrodiversity cellular system", IEEE Trans. on Vehic. Techn., Vol. 48 no.2, pp. 429-436, 1999.

[7] A. Conti, M. Z. Win, M. Chiani and J. Winters, "Bit error outage for diversity reception in shadowing environment", IEEE Commun. Letters., Vol. 7, no.1, pp. 15-17, 2003.

[8] A. A. Abu-Dayya and N. C. Beaulieu, "Micro- and macrodiversity MDPSK on shadowed frequency-selective channels", IEEE Trans. on Commun., 43(8), pp. 2334-2343, August 1995.
[9] S. H. Jamali and T. Le-Ngoc, Coded-Modulation Techniques for Fading Channels, Kluwer Academic Publishing, 1994. 